\title{
EVALUACIÓN DEL PROCESO DE IMPLEMENTACIÓN DE LA PLATAFORMA CLASSROOM EN LA UNAH 2020
}

\author{
EVALUATION OF THE IMPLEMENTATION PROCESS OF THE \\ CLASSROOM PLATFORM AT UNAH 2020
}

\author{
(Di) César Teófilo Zambrano Arce ${ }^{*}$, (D) Ketty Marilú Moscoso Paucarchuco ${ }^{1}$, (D) Mary Amelia \\ Cárdenas Bustamante ${ }^{1}$, (iD Rosa Cecilia González Ríos ${ }^{1}$, (D) Oseas Arístides Obregón Villantoy ${ }^{1}$ \\ czambrano@unah.edu.pe; kmoscoso@unah.edu.pe; mcardenas@unah.edu.pe; rgonzalez@unah.edu.pe; \\ oobregon@unah.edu.pe \\ ${ }^{1}$ Universidad Nacional autónoma de Huanta, Ayacucho, Perú
}

*Correspondencia: César Teófilo Zambrano Arce. Email: czambrano@unah.edu.pe

Recibido: 05.11.2020 | Aprobado: 12.12.2020

\section{RESUMEN}

El presente estudio se basa en el proceso de evaluación de la política pública universitaria de la educación a distancia y, de manera específica, la implementación de la plataforma Classroom, en sus dimensiones didáctica y operativa, en la Universidad Nacional Autónoma de Huanta (UNAH), periodo 2020, en el contexto de aislamiento físico y social, permitiendo un análisis acertado para la toma decisiones al respecto. Para medir tal impacto, se empleó el método científico no experimental, de nivel descriptivo; asimismo, la técnica de la encuesta, cuyo instrumento es el cuestionario, el cual fue aplicado a la población de la plana docente ordinaria de la institución.

Se enriquece los marcos conceptuales, exponiendo reflexiones a partir de una detallada revisión bibliográfica que analiza indicadores de contenidos, actividades, interactividad, recursos, administración y estética del ambiente virtual. Del análisis de las dimensiones resulta que un $90 \%$ promedio de docentes, ha realizado el proceso de implementación de la plataforma satisfactoriamente.

Palabras clave: Evaluación, procesos de implementación, plataforma Classroom, educación virtual.

\section{ABSTRACT}

This study is based on the evaluation process of the university public policy of distance education and, specifically, the implementation of the Classroom platform, in its didactic and operational dimensions, at the National Autonomous University of Huanta (UNAH), period 2020, in the context of physical and social isolation, which allowed an accurate analysis to make decisions in this regard. To measure such impact, the nonexperimental scientific method, descriptive level, was used; likewise, the survey technique, whose instrument is the questionnaire, which was applied to the population of the ordinary teaching staff of the institution.

The conceptual frameworks are enriched, exposing reflections from a detailed bibliographic review that analyzes indicators of content, activities, interactivity, resources, administration and aesthetics of the virtual environment. From the analysis of the dimensions, it appears that an average $90 \%$ of teachers have carried out the platform implementation process successfully.

Keywords: Evaluation, implementation processes, Classroom platform, virtual education. 


\section{INTRODUCCIÓN}

La sociedad está cambiando, aceleradamente, en estos momentos. Las transformaciones comunicativas han obligado a muchas de las instituciones a cambiar las relaciones de las estructuras políticas, sociales y económicas (Cabero et al., 2016, p.43); así es que, en lo que a educación se refiere, se ha aplicado estrategias inmediatas de comunicación sincrónica y asincrónica para el proceso de enseñanza - aprendizaje.

La crisis pandémica puso en evidencia la decadente situación en la que nos encontramos, resaltando sus debilidades no solo en salud, sino también en educación. De manera que, la presurosa decisión al cambio digital obligó a docentes y estudiantes a adoptar medidas de educación no presencial o virtual, términos que no discutiremos en esta ocasión.

La UNAH, como parte de su adaptación educativa, implementa la plataforma Classroom, la misma que se inició con una capacitación al estamento docente, de manera progresiva en el semestre 2020 - I, comenzando las actividades en agosto del mismo año.

La investigación parte del enfoque del docente, el cual, en un primer momento fue tradicionalista, donde el profesor es la figura central y es el principal responsable del aprendizaje del estudiante, ya que moldeado en un sistema cerrado y lineal es el encargado de la selección y diseño de lo que se aprende, y del cómo se aprende. Así mismo, la valoración de los logros estaba en función de los productos y la calidad de los mismos (Rodríguez \& Espinoza, 2017, p.1).

Por tanto, la UNAH, como la mayoría de las universidades públicas, centró su esfuerzo en mejorar las competencias digitales, a fin de perfilarse como una universidad innovadora y abierta a la tecnología. Ello significó el cambio de una pedagogía tradicional, donde los docentes utilizaban recursos como el plumón y la pizarra, sin tomar en cuenta que en las aulas universitarias ya estaban, entre sus estudiantes, los denominados millennials y la generación $Z$, individuos de la era digital, una generación que accede al mundo a través de tecnologías, son nativos digitales; es decir, resulta importante una adecuación a sus necesidades.

Por otro lado, para lograr satisfacción en los estudiantes se considera conveniente aplicar modelos que permitan un proceso de aprendizaje rápido, desarrollar la capacidad de realizar tareas simultáneas, sobre todo visuales, estar conectados por medios electrónicos, aprender haciendo, desarrollar la preferencia por la instrucción y construcción de su aprendizaje. En este marco cobra particular relevancia establecer modelos tecnológicos e innovadores de enseñanza.

Bajo esas circunstancias se plantea la siguiente interrogante ¿Se desarrolló de manera satisfactoria el proceso de implementación de la plataforma Classroom? la respuesta contribuirá a mejorar la calidad educativa universitaria desde la arista de la educación virtual. Su carácter científico contribuye a las bases teóricas de sus dimensiones, mientras a nivel práctico define si es favorable su implementación; para la medición de la variable, la metodología fue consistente y objetiva. 


\section{MATERIALES Y MÉTODOS}

Esta investigación es cuantitativa, descriptiva, puesto que busca evaluar el proceso de implementación de la plataforma Classroom, durante el periodo de inmovilización obligatoria provocada por la pandemia. Se analizó a la población integrada por los docentes ordinarios de la UNAH, con una muestra representativa cuyo margen de error fue del 5\%, con un nivel de confianza del $95 \%$, equivalente a 20 docentes. El diseño de la investigación no experimental se basó en el estudio de la variable implementación de la plataforma Classroom, las dimensiones son el aspecto didáctico y el aspecto operativo del ambiente virtual, cuyos indicadores se plasman en la siguiente tabla:

\section{Tabla 1}

Dimensiones e indicadores de la variable

\begin{tabular}{lll}
\hline Variable & Dimensión & Indicadores \\
\hline & Aspectos didácticos del & Contenidos \\
& ambiente virtual & Actividades \\
Implementación de plataforma & & Interactividad \\
Classroom & Aspectos operativos del & Recursos \\
& ambiente virtual & Administración \\
& & Estética \\
\hline
\end{tabular}

La investigación se desarrolló mediante la técnica de la encuesta, cuyo instrumento fue un cuestionario de 30 preguntas, considerando 05 preguntas para cada indicador. Su aplicación se llevó a cabo mediante el formulario de Google, con el apoyo del área administrativa de la institución.

\section{PANORAMA ACTUAL DE LA EDUCACIÓN VIRTUAL UNIVERSITARIA}

La pandemia exigió el abandono de la educación convencional e impuso retos a docentes y estudiantes de las diversas universidades públicas, quienes de manera acelerada tuvieron que adaptarse a la educación virtual, incluyendo de manera transversal a las tecnologías de información y comunicación. Todas las personas son conscientes del cambio exponencial que representa el uso de la tecnología en la vida cotidiana, sobre todo los jóvenes que dedican la mayor parte del día en el uso de aparatos electrónicos, convirtiéndolos en nativos digitales. Esto se debe principalmente al modo de cómo los jóvenes obtienen información relevante, válida y confiable; que se ratifica por medio de conferencias académicas en diversas plataformas como el Meet, Zoom y otros (Samper et al., 2017, p.1).

Por otro lado, la evolución de las TIC exige al docente universitario dejar de ser un simple expositor y complementar sus labores como administrador, ingeniero, técnico y gestor del conocimiento, facilitando la comunicación con sus estudiantes.

La revolución comenzada, décadas atrás, hoy, gracias a esta pandemia, logra consolidarse en el quehacer pedagógico. A la inversa de la educación que recibimos, aquellos que nos educamos a fines del siglo XX o comienzos del XXI, el estudiante ya no se acomoda a un plan de estudio rígido, hoy decide que aprender; en cambio, el docente solo presenta las intenciones del curso y sus competencias. Es lo que Aparicio (2004, 
citado por Paéz) denomina modelo horizontal de comunicación. Progresivamente, a medida que se complica la adquisición de conocimientos, se necesitará del guía, mediador, que será el responsable de conducir al estudiante a la adquisición de las competencias, así como destrezas y valores propios que se relacionan con la Era de la Información ( Páez, H. \& Arriaza, E., 2005, p. 5).

El aprendizaje activo está determinado por el uso de las tecnologías; de modo que, quien no use softwares, tiene desventajas frente al resto; gestionar los entornos virtuales involucra resolver problemas en conjunto, corregir los errores cometidos permanentemente, que los estudiantes pongan de manifiesto sus dudas, dificultades y limitaciones, generando ocasión propicia para que el catedrático, en su condición de experto, logre desarrollar las competencias empleando el método socrático. En esta condición, las TIC brindan una mayor oportunidad para que el docente atienda las diferencias individuales y a la vez integre al grupo ( Páez, H. \& Arriaza, E., 2005, p.5).

En este contexto, hay quienes se entusiasman con la desaparición del aula, y con ello todo lo que involucra tener ese espacio físico. Las instituciones permanecerán a pesar de estas nuevas realidades, comprendiendo que los aprendizajes sobrepasan la capacidad humana y que son susceptibles de ser asistidos por los medios tecnológicos, con lo cual se acrecienta la inteligencia humana. En consecuencia, el desafío para la comunidad universitaria es utilizar todas las tecnologías disponibles para crear en ellas entornos que propicien el desarrollo de sus estudiantes que combinen capacidades para utilizar los vastos recursos tecnológicos en su crecimiento intelectual, así como la expansión de sus habilidades; de manera que se motive a los estudiantes a estar comprometidos con su propio aprendizaje ( Páez , H. y Arriaza, E., 2005, p.7).

\section{PLATAFORMA CLASSROOM}

Es muy útil el empleo del Classroom, ya que gracias a esta plataforma se comparte todo tipo de documento, se organiza el aprendizaje en carpetas y sobre todo permite la comprensión de los objetivos y competencias que busca el docente en el perfil del estudiante universitario. En ese sentido, (INNEDU, 2020) conocedor de temas educativos, en su portal web considera que el Classroom es una herramienta que "permite gestionar el aprendizaje a distancia o mixto (semi presencial)".

Google Classroom es un Entorno Virtual de Aprendizaje (EVA), se administra bajo la patente de google educativo. Es beneficioso para los estudiantes y docentes, porque supera los límites de la disciplina clásica, los usuarios son notificados en tiempo real, facilitando los procesos de retroalimentación; Alves y Lima (2018, p. 590) señalan que: "Esta plataforma simplifica la creación y organización de tareas, el feedback en tiempo real entre otras características y funcionalidades".

Si bien es cierto, el Classroom alienta una comunicación directa y en tiempo real, entre el docente y estudiante; los archivos y mensajes pueden ser enviados con anterioridad; las evaluaciones pueden programarse, considerándose una fortaleza para esta herramienta. El Classroom relaciona a los estudiantes con los contenidos de la materia, el docente puede enviar un archivo en distintos procesadores, libros con licencia o de código abierto; asemejándose a un red social de carácter académico, donde hay foros, debates, 
videoconferencias, lo que permite evaluar y hacer un seguimiento del estudiante en relación a sus competencias y a los objetivos propuestos por el sistema educativo (Moya Fuentes et al., 2017, p. 2569).

Para (Basso-Aránguiz et al., 2018, p.2), los modelos Classroom se presentan como una propuesta tecnológica de recursos digitales gratuitos para asignaturas de distintos niveles de formación en educación, creando una experiencia que puede ser replicada en cualquier institución, que quiera innovar su proceso de enseñanza-aprendizaje, independiente de su presupuesto en inversión tecnológica.

Google es una compañía bien posicionada en el mercado, su motor de búsqueda es rápida y confiable, brindando comodidad a los usuarios desde sus hogares, mediante el uso de teléfonos móviles, computadoras portátiles y otros. Esta empresa promociona su propio sistema operativo (S.O.) llamado Android, posicionándose de manera sólida en el mercado.

(Acosta et al., 2017, p. 8) señalan que: “Google ha desarrollado una aplicación, la cual es una herramienta para facilitar la enseñanza y el aprendizaje, esta se llama Classroom, el profesor va creando la estructura de la clase y la organiza adecuando los temas y tiempos de manera propicia". Desde la experiencia de la UNAH, esta herramienta complementa la actividad docente, hace posible el desarrollo de las clases en condiciones de confinamiento y la distancia social, convirtiendo la virtualidad en una oportunidad de construcción de aprendizaje, a través del uso de simuladores, software, aplicaciones, etc. para los diversos talleres y práctica que se dan en el proceso educativo.

\section{Implementación de la plataforma Classroom}

La coherencia entre la planificación del syllabus y la pedagogía debe estar en relación con el pensamiento crítico que podamos desarrollar en nuestros estudiantes; de manera que, analicen la información publicada en redes o libros, cuestionando su veracidad y calidad, potenciando el sentido de la razón, reflexionando sobre los avances de su aprendizaje, y sobre todo conservando la autonomía de sus ideas. Es necesario que el estudiante tenga un correo institucional, que facilite la administración desde los sistemas de gestión de aprendizaje (SGA), accediendo de manera cómoda y fácil al aula virtual donde se imparten sus asignaturas (Tarango et al., 2007, p.1).

Según Tellez (2014), mencionado por Tarango (2007, p.1.), el ambiente de aprendizaje adecuado permite el desarrollo de capacidades y habilidades en los estudiantes. Con el ingreso de las TIC se puede observar diferencias en la disponibilidad de recursos, para desempeñarse en el quehacer educativo, se necesita de una adecuada implementación, basada en inversión pública y respaldada por el sistema de gestión de aprendizaje (SGA). Por lo tanto, el Estado debe adoptar políticas de inversión educativa, que favorezcan a estudiantes y docentes, como capacitaciones, educación transversal en TIC en los diferentes niveles, implementación de equipos tecnológicos, adquisición de dispositivos móviles, redes de internet, entre otros.

Por su parte la UNAH, realizó capacitaciones a sus docentes y estudiantes en el uso de la plataforma Classroom, distribuyó chips con líneas de internet, facilitó el uso de laboratorios de computación; pese a ello, su esfuerzo no cubre las necesidades en relación 
a recursos tecnológicos y conectividad, considerados como un problema nacional, que se evidenció a raíz de la pandemia.

\section{Aspectos didácticos del ambiente virtual}

El mundo gira en la gestión de las tecnologías, las instituciones y empresas se comunican con sus usuarios, lo mismo hace la universidad, la institución genera conocimiento, ciencia y tecnología. La sociedad se volvió, de un día para otro, en una sociedad sujeta a la red, los ciudadanos descubren que hay maneras de comunicarse, y todos quieren aprender, hemos pasado a la sociedad educadora. Surge una pregunta a la racionalidad pedagógica ¿Cómo se adecua el docente al proceso de enseñanza aprendizaje en este nuevo contexto?

El SGA es una gran herramienta, que requiere de información específica, prestándole especial atención a los perfiles de los estudiantes, como un elemento fundamental en el logro de resultados. En la práctica docente se recomienda desarrollar la metacognición y la reflexión en los estudiantes, sin generar dependencia en las TIC, por el contrario, considerarlo como herramienta de carácter significativo en la educación. Las TIC, nos gusten o no, ya se instalaron en la educación universitaria, antes era un privilegio de aquellos que estudiaban en la modalidad a distancia o semipresencial, hoy, en tiempos de pandemia, es normal observar a los docentes interactuar en las comunidades virtuales; se proyecta esta realidad de manera permanente en el futuro.

Los entornos virtuales diseñados de manera multidisciplinar, hoy están incorporados a la pedagogía universitaria; es responsabilidad de los actores académicos familiarizarse con estos entornos. El impacto de las tecnologías de la información nos llama a la reflexión, no sólo a los docentes de informática o jefes de laboratorio de cómputo, sino también a todos los docentes que laboran en la universidad. La pedagogía de la virtualidad, herramienta del presente, es necesaria en el quehacer normal de correlacionar pedagogía e informática, así como de las redes sociales para un aprendizaje mayor. En ese razonamiento, es importante tener en cuenta que la mediación cognitiva tiene como función fundamental el aprendizaje. Es necesario comprender los esquemas cognitivos que interactúan para modificarse y aprender, también la forma de evaluar formativamente. Se ha corroborado que esta mediación pedagógica se da por vía electrónica a través de mensajes del Google Meet, WhatsApp, correos electrónicos o mensajes de texto, también se da una interacción unilateral entre el estudiante y el autor de un texto donde el docente puede intervenir para reforzar contenidos no entendidos. Mediante la lectura, el estudiante se pone en contacto con los esquemas cognitivos del autor que pueden empalmar con el apoyo del catedrático. Esta relación también es posible entre el aprendiz y sus pares que discuten en un foro acerca de alguna problemática.

\section{Contenido}

Durante esta crisis sanitaria, en situación de confinamiento, se ha demostrado que las redes informáticas y el uso correcto de los aplicativos desaparece la necesidad de coincidir en el espacio y en el tiempo; esto trae consecuencias importantes para la educación superior y lo que ella representa. La interacción es muy fluida, dependiendo de la velocidad del internet y los equipos tecnológicos, los estudiantes y maestros comparten información, pero solo los últimos imparten formación. Una característica resaltante es 
que el docente ya no es el centro de atención, la educación está descentralizada y es periférica con una masa de espectadores que gestionan tecnología y crean comunidades virtuales que han decidido invadir espacios en las redes. Estos nuevos entornos de enseñanza-aprendizaje están basados en el aprendizaje cooperativo y colaborativo, bajo la lógica de Vigotsky ( Páez H. y Arriaza, E., 2005, p.8).

\section{Actividades}

Encontramos que la World Wide Web (WWW) nos comunica con el mundo, en especial a la comunidad científica que se encuentra en las universidades, es innegable que los tutoriales ayudan a la creación de nuevos portales alternativos y algunos antisistemas, así como nuevos escenarios web, favoreciendo enormemente los entornos de aprendizaje. No es novedad que los propios estudiantes y profesionales se organicen desde cualquier rincón del mundo para realizar un conversatorio o dar a conocer algún gran descubrimiento, en especial los estudiantes de la UNAH, que han podido interactuar con sus pares universitarios del mundo e incluso suscribiendo pronunciamientos sobre situaciones tan delicadas para la comunidad científica como la vacuna obligatoria o las prácticas bioéticas; los docentes no quedan al margen, están en esa búsqueda de nuevos aprendizajes, también buscan involucrarse en la compra de software o aplicativos para sus sesiones; hoy muchos docentes lucen sus potentes procesadores, lo que antes bien podría ser un auto del año (Sánchez y Salvador, 2010, p.163).

\section{Interactividad}

Luego de la revolución agraria, en siglos posteriores, las revoluciones industriales solo aceleraban la producción en serie. No obstante, el teléfono en 1876 apertura algo que no era necesario aún, el cual fue utilizado en la Segunda Guerra Mundial, nos referimos al internet, hasta volverse comercial en 1983. Hoy un celular con internet es el mundo en nuestras manos, el sujeto interactúa con un dispositivo desde cualquier parte del mundo, claro con algunas restricciones en Asia y otros países, una libertad indiscutida para nuestra realidad, solo que los planes son caros para muchos estudiantes, quienes no pueden sostener ese presupuesto por varios meses ( Páez, H. \& Arriaza, E., 2005, p.5).

\section{Aspectos operativos del ambiente virtual}

En los ambientes virtuales encontramos que las TIC se utilizan en provecho de la educación, y que estás a su vez modifican la educación tal y cual la hemos venido entendiendo hasta antes de la pandemia. Las TIC y los aplicativos educativos no encontraron mejor escenario que presentarse en esta crisis y permanecer en la educación universitaria, y con esto no estamos resumiendo a un centro de cómputo sino a una realidad que se lleva en los bolsos y bolsillo, el celular. Desde un celular se puede aprender mucho, desde una tableta más, pero desde un computador debe ser el infinito, por su capacidad de almacenamiento (Sánchez y Salvador, 2010, p.165).

Observar todas estas características en la universidad nos acerca a docentes de distintas especialidades que pueden analizar el fenómeno con otra óptica y perfilarlo como un caso complejo en la perspectiva de Edgar Morín. El docente universitario será un pedagogo de la complejidad de los sistemas, sin necesidad de asistir a cursos relacionados al tema; la pandemia los ha obligado a autoeducarse en TIC, que en realidades distintas tomaría años aprender. 


\section{Recursos}

Quien no sepa utilizar una computadora o cualquier dispositivo electrónico es considerado el nuevo analfabeto del siglo XXI. Ahora podemos afirmar que un docente debe incorporar su computadora personal o su celular a sus actividades académicas, disponer de software idóneo; la capacitación la encuentra en el YouTube, el sentido común lo debe acompañar para crear las aulas y sobre todo ordenar la información, para tener una gran paciencia y no sentirse incómodo cuando un estudiante le hace presente que encontró algo en redes que entra en conflicto con lo que él le enseñó (Sánchez y Salvador, 2010, p.164).

En esta pandemia surgieron los grandes teóricos de la educación virtual, los aportes no sobran, son necesarios porque explican, desde el quehacer pedagógico, la situación de crisis sanitaria hasta la luz de esperanza con los entornos virtuales, clasificando como un punto de partida para posteriores investigaciones y convertirlas en recomendaciones de políticas públicas.

La realidad pandémica ha limitado a la comunidad universitaria, al acceso de conectividad, responsabilidad de gobierno central, que en los últimos tiempos se ha visto envuelto en actos de corrupción, limitando el desarrollo de la educación; por diversas adquisiciones de equipos de cómputo a altos precios y con tecnología obsoleta.

\section{Administración}

La administración en su totalidad es controlada por UNAH, a través de sus especialistas en entrono virtual, de manera externa se encuentra a cargo de la Oficina de Tecnología de Comunicación e Información, quienes se encargan de la grabación de las sesiones y su publicación en plataforma, los docentes administran de manera interna sus materiales, distribuyéndolos en semanas de sesiones sincrónicas y asincrónicas.

\section{Estética}

Es la forma como visualizan los estudiantes los materiales generados por el docente, que consiste en Ppts, videos, lecturas, foros; esta visita debe ser amigable y fácil de usar. La UNAH ha estructurado su diseño de manera conjunta iniciando en la pestaña del tablón, donde se brinda la bienvenida y presentación al curso, indicando el horario de las sesiones sincrónica y asincrónica, así con el enlace al Meet, siguiendo la pestaña de trabajo de clase, en la cual se ha sistematizado 16 sesiones distribuidas en semanas, donde se detalla las indicaciones, se publican los materiales y tareas; en la pestaña de personas, se administra la cantidad de docentes y estudiantes, accediendo a una comunicación a través de correos electrónicos institucionales; en la pestaña de calificaciones, se encuentra el reporte de actividades y tareas entregadas, las mismas que son evaluadas cuantitativamente por el sistema de acuerdo a la programación desarrollada por el docente.

\section{Evaluación del proceso de implementación de la plataforma Classroom}

Las TIC nos permiten interactuar con los estudiantes, pero no podemos conocer ni comprender totalmente el aspecto subjetivo en relación a su carácter y sentimientos. El SGA no hace más que registrar datos del curso, el enlace del Meet, el registro de las notas, etc.; el rol del maestro es importante porque se relaciona de manera emotiva con el estudiante lo que le permite evaluar otros aspectos como el esfuerzo, progreso y 
perseverancia, factores relevantes en el sistema de evaluación, que coadyuva al desarrollo de habilidades, capacidades y finalmente competencias (Tarango et al., 2007, p.2.).

La evaluación de los aprendizajes no debe resumirse obtener notas para que el estudiante pase al siguiente nivel, evaluar es emitir un juicio sobre el desempeño, pero esta no puede ser sumativa, tiene que ser formativa, que se enfoque en obtener información cualitativa de interpretación que sirva para lograr los objetivos y capacidades que persigue el syllabus.

El área académica de la universidad se encarga de evaluar el sistema de educación a distancia, debe crear instrumentos idóneos para recolectar información válida, que mejore el entorno virtual en la dualidad docente estudiante como actores importantes en el quehacer pedagógico de la UNAH. Hay que poner en claro que virtualizar la educación superior implica un cambio en la modalidad, que debe respetar el aspecto epistemológico y pedagógico de la educación superior. Para evaluar la educación virtual hay que reflexionar, analizar los paradigmas pedagógicos, variables como (hardware y software) recursos tecnológicos, posibilidad de acceso a internet (Maenza, 2006, p.3).

Hace 50 años nadie se hubiera imaginado lo fantástico y lo increíble que se puede hacer, desde un rincón de la casa, con una computadora o cualquier otro dispositivo móvil con un microprocesador y memoria que constan de infinidad de softwares con utilidades determinadas, como juegos, reproductores de música, instructivos de todo tipo, también se realizan transacciones bancarias, servicios de taxi, delivery; lo más resaltante es que se puede acceder y profundizar el conocimiento humano en áreas como la ingeniería, estadísticas, entre otras; por su parte la universidad adquirió e implementó softwares, para un mejor proceso educativo.

La evaluación educacional es un reto compartido entre los actores donde se busca el desarrollo de competencias, Maenza (2006) menciona que "Los alumnos pueden realizar actividades más complejas, trabajando progresivamente para la elaboración de un reporte o un proyecto, efectuados en forma individual o grupal", lo que significa que el trabajo colaborativo entre estudiantes brinda resultados favorables y fortalece la relación entre pares, creando un sentido crítico, reflexivo y autónomo.

En cuanto a la evaluación del uso de la tecnología, hay criterios definidos como la utilidad y la operacionalidad del recurso. La evaluación en este sentido tiene que ver con el análisis del ambiente referente al sujeto de aprendizaje. Si un alumno ha logrado incorporar conocimientos después de emplear el uso de la herramienta, se entiende que el sistema ha logrado el objetivo; este en algunos casos se puede desarrollar de manera personalizada. En cuanto a los programas desarrollados dentro de ambientes virtuales, se pueden destacar la comunicación, navegación, acompañamiento, cooperación, la investigación formativa y tutoría.

Los aplicativos educativos y el rendimiento del estudiante guardan relación directamente proporcional, no existe uno sin lo otro. En caso que el estudiante no haya podido lograr las competencias planificadas en el tiempo previsto, los aplicativos tendrán que ser sustituidos. El docente, en la política de mejoras, tiene que buscar aplicativos 
idóneos donde la virtualidad se asocie a las reformas de calidad, para comprobar ello es necesario aplicar cuestionarios de satisfacción a los estudiantes.

El docente realiza la reingeniería de su propio trabajo, esto se facilita con un estudiante motivado, así mismo, debe contar con un plan de reforzamiento periódico, compartir resultados con sus estudiantes y juntos, planificar las políticas de mejora. Escuchar al estudiante ya es un cambio de paradigma en la educación universitaria, su opinión importa, es una referencia que tomamos como punto de partida, la disertación cede paso a la estrategia de entornos virtuales para saber cómo aprenden, cómo están repasando los textos, audios y videos; valorando la intervención como docentes en la construcción del conocimiento (Fernández et al., 2017, p.1).

\section{RESULTADOS Y DISCUSIÓN}

\section{Dimensión aspectos didácticos del ambiente virtual}

En la siguiente secuencia de gráficos se ilustra los resultados obtenidos en la encuesta aplicada a los docentes de la UNAH, la sistematización de las mismas se realizó tomando en cuenta los ítems de los indicadores correspondientes a cada una de las dimensiones consideradas en la investigación.

\section{Indicador contenido}

\section{Gráfico 2}

Aspectos didácticos del ambiente virtual: Contenidos

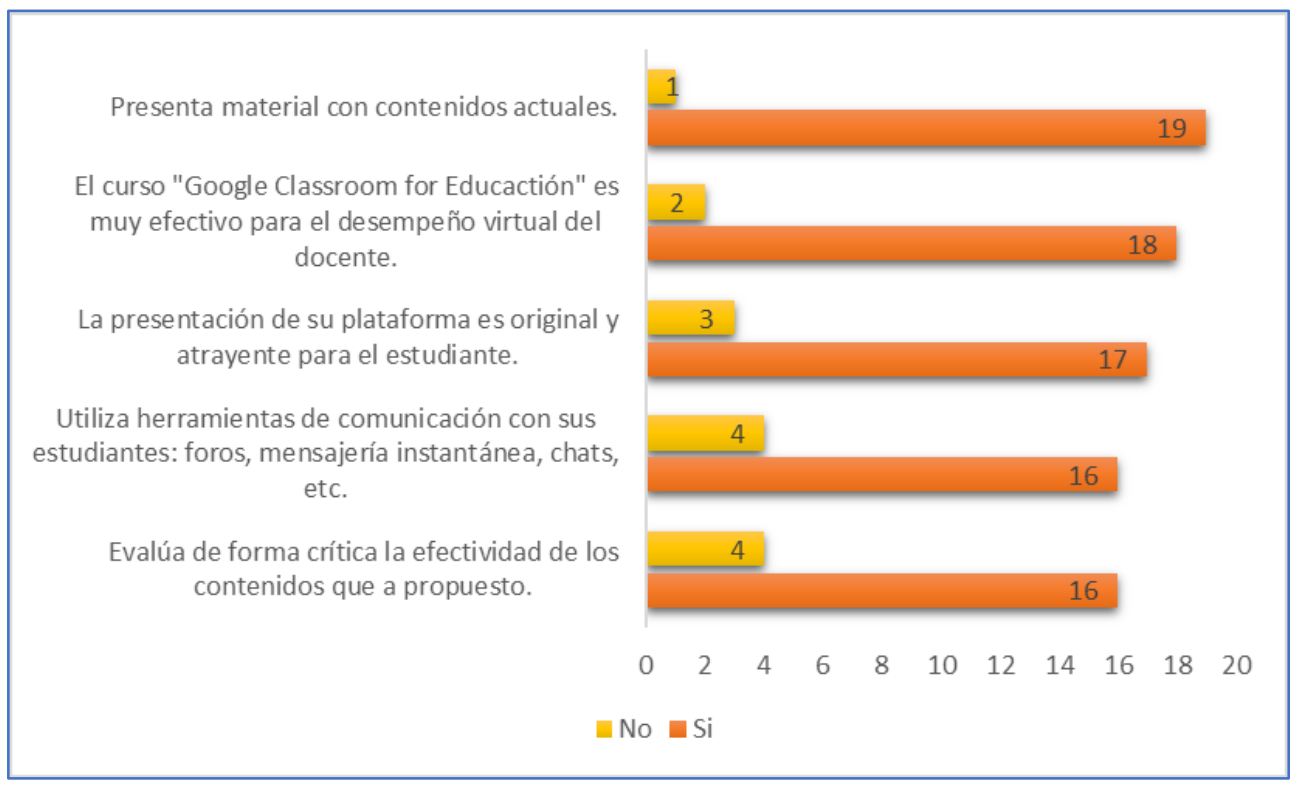

De acuerdo a los resultados determinados en el Gráfico 1, sobre los aspectos didácticos del ambiente virtual: en el ítem de Contenidos, se encontró que el 95\% de docentes presenta materiales actualizados, el 90\% considera el curso de capacitación muy efectivo en el desempeño virtual del docente, el $85 \%$ cree que la presentación de su plataforma es original y atrayente para el estudiante, el 80\% utiliza herramientas de comunicación, como foros, mensajería instantánea, chats, etc y evalúa de forma crítica la efectividad de los contenidos propuestos. 


\section{Gráfico 2}

Indicador Actividad

Aspectos didácticos del ambiente virtual: Actividades

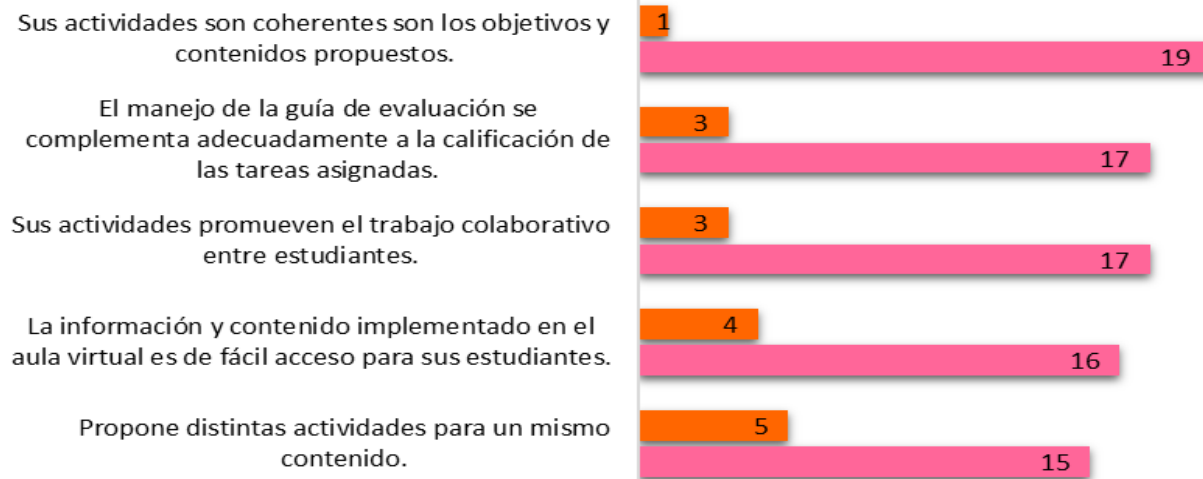

El manejo de la guía de evaluación se complementa adecuadamente a la calificación de las tareas asignadas.

Sus actividades promueven el trabajo colaborativo entre estudiantes.

La información y contenido implementado en el aula virtual es de fácil acceso para sus estudiantes.

Propone distintas actividades para un mismo contenido.

De acuerdo a los resultados determinados en el Gráfico 2, sobre los aspectos didácticos del ambiente virtual: en el ítem de Actividades, el 95\% considera que sus actividades son coherentes con los objetivos y contenidos propuestos, el $85 \%$ maneja la guía de evaluación adecuadamente para la calificación de tareas y promueve el trabajo colaborativo entre sus estudiantes, el 80\% muestra una información y contenido de fácil acceso, mientras que el $75 \%$ promueve distintas actividades para un mismo contenido.

\section{Indicador Interactividad}

\section{Gráfico 3}

Aspectos didácticos del ambiente virtual: Interactividad

Refuerza sus actividades académicas con otras herramientas digitales para mejorar el aprendizaje de su estudiante.

La plataforma resulta útil para que el estudiante aprenda un contenido determinado.

La plataforma presenta facilidad y disponibilidad de uso.

La plataforma es repetitiva.

Se siente autónomo en el uso de la plataforma.
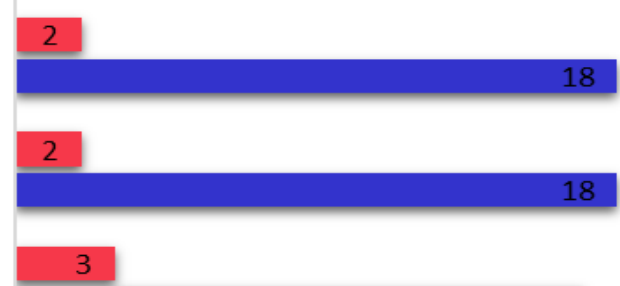

\section{3}

\section{5}
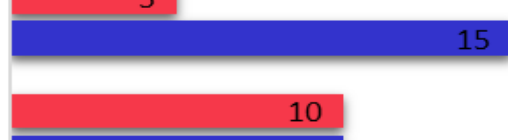

$\begin{array}{llllllllll}2 & 4 & 6 & 8 & 10 & 12 & 14 & 16 & 18 & 20\end{array}$

No $\square \mathrm{Si}$

De acuerdo a los resultados determinados en el Gráfico 3, sobre los aspectos didácticos del ambiente virtual: ítem de Interactividad, se encontró que el 90\% de los docentes refuerza sus actividades académicas con otras herramientas digitales para mejorar el aprendizaje y la plataforma resulta útil en el aprendizaje, el 85\% afirma que la 
plataforma está disponible y es de fácil uso, el $75 \%$ de docentes considera a la plataforma como repetitiva y el 50\% no se siente autónomo con el uso de la plataforma.

\section{Dimensión aspectos operativos del ambiente virtual}

\section{Indicador Recursos}

\section{Gráfico 4}

Aspectos operativos del ambiente virtual: Recursos

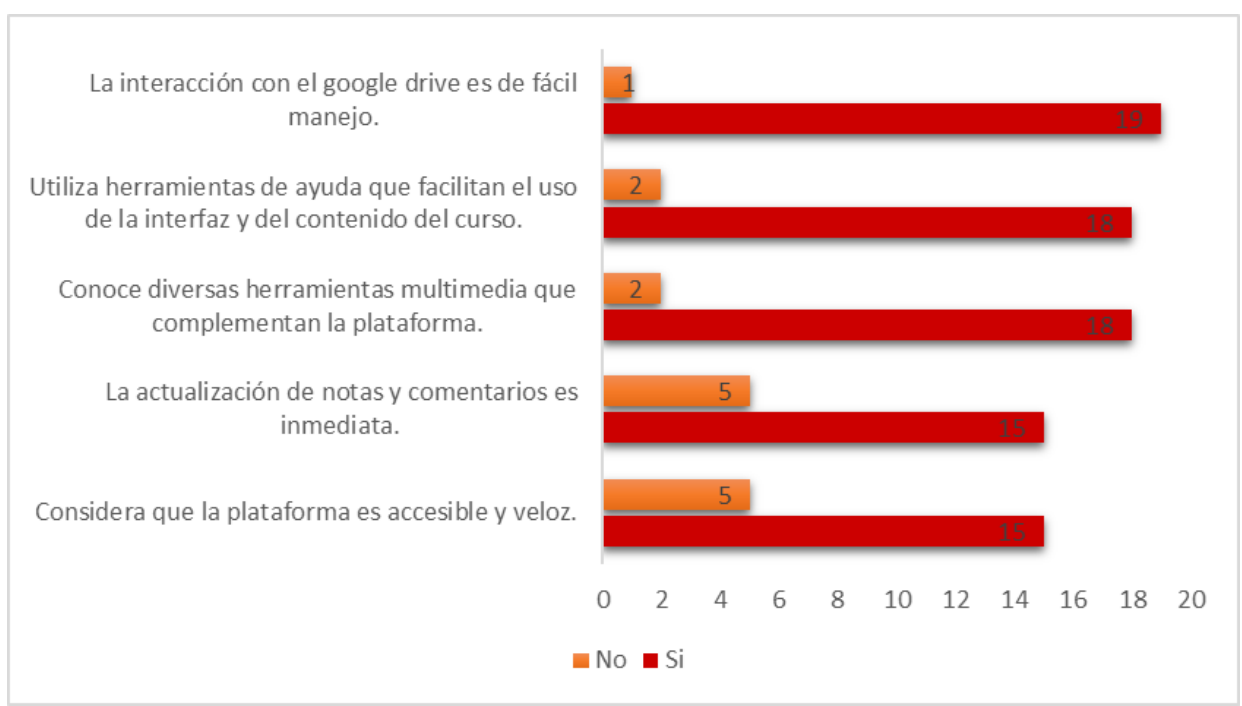

De acuerdo a los resultados determinados en el Gráfico 4, sobre los aspectos operativos del ambiente virtual: ítem de Recursos, al 95\% de docentes le resulta fácil la interacción con el google drive, el 90\% utiliza herramientas de ayuda que facilitan el uso de la interfaz y del contenido del curso, así como conoce diversas herramientas multimedia que complementan la plataforma, el $75 \%$ actualiza las notas y comenta de manera inmediata y también consideran que la plataforma es accesible y veloz.

\section{Indicador Administración}

\section{Gráfico 5}

Aspectos operativos del ambiente virtual: Administración

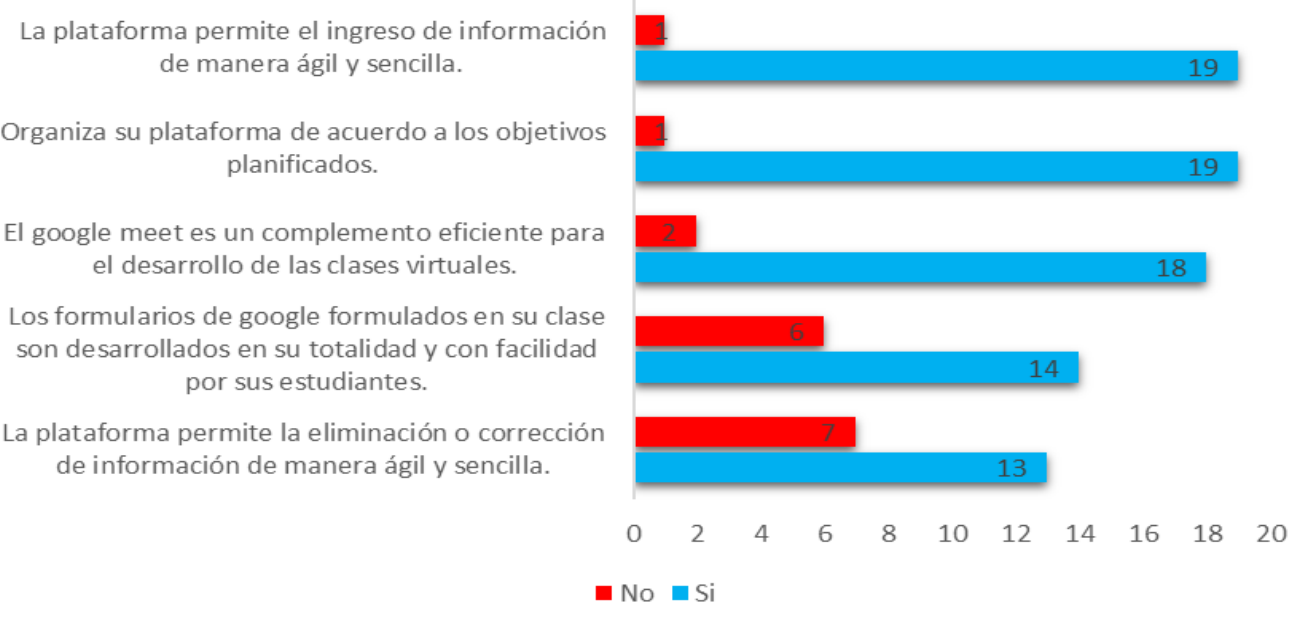


De acuerdo a los resultados determinados en el Gráfico 5, sobre los aspectos operativos del ambiente virtual: ítem de Administración, el 95\% de docentes considera que la plataforma permite el ingreso de información de manera ágil y sencilla, como también organiza su plataforma de acuerdo a los objetivos planificados, el $90 \%$ asegura que el google meet es un complemento eficiente para el desarrollo de sus clases virtuales, el 70\% genera formularios de google con facilidad y al 65\% le resulta fácil la eliminación o corrección de la información.

\section{Indicador Estética}

\section{Gráfico 6}

Aspectos operativos del ambiente virtual: Estética

El interfaz genera un ambiente llamativo para el estudiante.

La arquitectura didáctica del aula virtual facilita el desarrollo interactivo de las clases.

Usa mensajes variados: informativos, identificatorios, expresivos, normativos e imperativos.

La plataforma reproduce videos y la sincronización es de calidad.

Considera que la plataforma presenta de manera óptima la utilización del audio, imágenes estáticas y en movimiento, animación.

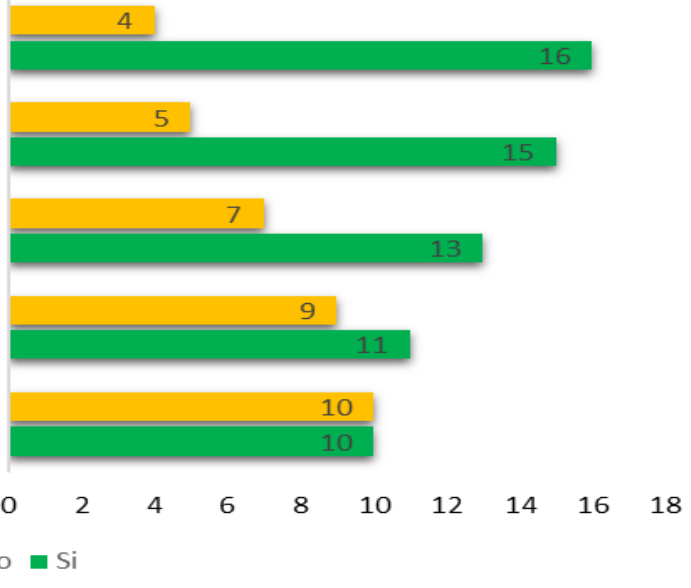

De acuerdo a los resultados determinados en el Gráfico 6, sobre los Aspectos operativos del ambiente virtual: ítem de Estética, el 80\% opina que el interfaz genera un ambiente llamativo para el estudiante, el 75\% estima que la arquitectura didáctica facilita el desarrollo interactivo de las clases, el $65 \%$ usa mensajes variado (informativos, identificatorios, expresivos, normativos e imperativos), el 55\% reproduce videos con sincronización de calidad y el 50\% considera que la plataforma representa de manera óptima la utilización de audio, imágenes estáticas y en movimiento (animación).

\section{CONCLUSIONES}

- El uso del Classroom y las herramientas del Google educativo permiten alcanzar las competencias planteadas en cada asignatura; su diseño sencillo no necesita de algún software, ya que el estudiante se familiariza fácilmente.

- Todas las actividades se pueden archivar, incluidos libros, videos, audios, etc. así como el avance de las tareas en el Google Drive, y lo más importante es la funcionalidad de esta plataforma que permite el uso de dispositivos móviles.

- La sistematización y la organización que ofrecen los docentes se basa en el sílabo de la asignatura; esta se desarrolla teniendo en cuenta el cronograma de actividades, logrando una mejor gestión de la información y comunicación entre estudiantes y docentes. 
- La emergencia sanitaria ha promovido la especialización de los docentes en el uso de las herramientas digitales, siendo los principales beneficiarios los estudiantes.

- El portafolio de actividades, es flexible porque se presenta en tiempo real, es rápido, además permite publicar, editar, descargar y borrar información.

- Los beneficios de la educación virtual son: la optimización del tiempo en el desarrollo de clases, acorta distancias, ahorro en el uso del papel, bajo costo en la adquisición de libros digitales, información complementaria en línea, interactividad académica, entre otros.

- El acceso a diferentes materiales informativos genera la democratización e igualdad entre los estudiantes, acortando brechas sociales y económicas.

- Del resultado estadístico de las variables de evaluación en la implementación de la plataforma Classroom, aspectos operativos y didácticos, se puede manifestar que el estamento docente, implementó de manera positiva los recursos y materiales, asumiendo con motivación el traslado de la educación tradicional a la virtual.

- Del análisis de las dimensiones en términos porcentuales, más del 90\% ha realizado el proceso de implementación de la plataforma Classroom satisfactoriamente. Queda pendiente realizar un análisis a nivel del estamento estudiantil.

\section{REFERENCIAS BIBLIOGRÁFICAS}

Acosta, N. K. M., Cortes, R. M. P., \& Maldonado, J. A. S. (2017). Ambientes Educativos a Distancia para la Mejora de la Enseñanza: Uso de Classroom. Revista Electrónica Sobre Cuerpos Académicos y Grupos de Investigación, 4(0), 1-13.

Alves, F. B., \& Lima, D. A. (2018). Uso de la clasificación para el análisis y la minería de datos en la herramienta de enseñanza-aprendizaje Google Classroom. Nuevas

Basso-Aránguiz, M., Bravo-Molina, M., Castro-Riquelme, A., \& Moraga-Contreras, C. (2018). Propuesta De Modelo Tecnológico Para Flipped Classroom. Revista Electrónica Educare, 22(2), 1-17. https://doi.org/10.15359/ree.22-2.2

Cabero Almenara, J. C., \& Lopéz Meneses, E. L. \& Jaen Martínez Alicia (2016). Los portafolios educativos virtuales en las aulas universitarias. Instrumentos didácticos para la innovación docente y la calidad de los procesos de enseñanza y. 5374, 43-70.

Fernández, J. M., Carrasco, C. J. G., \& Pedro Miralles Martínez. (2017). Evaluación del diseño e implementación de la metodología Flipped-classroom en la formación del profesorado de Ciencias Sociales. Revista de Educación a Distancia, 0(0), 1-26.

Páez H. \& E. Arriaza. (2005). Uso de una plataforma virtual de aprendizaje en educación superior. Caso nicenet.org. Paradigma, 26.

Innedu (2020). https://www.innedu.es/bootcamps/google-classroom-que-es-y-paraque-sirve/ Consultado el 04 de octubre 2020.

Maenza, R. R. (2006). Indicadores de evaluación para plataformas virtuales empleadas en educación. August. https://doi.org/10.13140/RG.2.1.3545.8804 
Moya Fuentes, M., Bia Platas, A., Carrasco Andrino, M., Jiménez Pascual, M., Ramón Martín, A., \& Soler García, C. (2017). 3779_Memoria Red en metodologías docentes con TICS 2016/2017: implementación de la plataforma virtual Google Classroom. Memorias Del Programa de Redes-I 3 CE De Calidad, Innovación e Investigación En Docencia Universitaria . Convocatoria 2016-17, 2564-2574.

Rodríguez Zamora, R., \& Espinoza Núñez, L. A. (2017). Trabajo colaborativo y estrategias de aprendizaje en entornos virtuales en jóvenes universitarios. RIDE Revista Iberoamericana para la Investigación y el Desarrollo Educativo, 7(14), 103. https://doi.org/10.23913/ride.v7i14.274

Samper, M. D., Garcia-Garcia, D., Ferri, J. M., Carbonell-Verdu, A., \& López Martínez, J. (2017). Implementación de la plataforma GOOGLE CLASSROOM en la asignatura "Tratamiento de Residuos" para la realización de experiencia de clase inversa. July. https://doi.org/10.4995/inred2017.2017.6818

Sánchez, A. B., \& Salvador, C. C. (2010). Los entornos virtuales como espacios de enseñanza y aprendizaje. Revista Mexicana de Investigación Educativa, 15(0), 163-184.

Tarango, J., Machin-mastromatteo, J. D., \& D, M. J. (2007). Aprendizaje de Google classroom y evaluación según diseño y aprendizaje de Google classroom y chamilo design and learning evaluation of google classroom and chamilo. 10(19), 91-104.

\section{CITAR COMO:}

Zambrano Arce, C. T., Moscoso Paucarchuco, K. M., Cárdenas Bustamante, M. A., González Ríos, R. C., \& Obregón Villantoy, O. A. (2021). Evaluación del proceso de implementación de la plataforma classroom en la UNAH 2020. Puriq, 3(1), 136-150. https:// doi.org/10.37073/puriq.3.1.161 